\title{
Rapamycin-resistant and torin-sensitive mTOR signaling promotes the survival and proliferation of leukemic cells
}

\author{
Seohyun Park, Hyunsub Sim \& Keunwook Lee * \\ Department of Biomedical Science, College of Natural Science, Hallym University, Chuncheon 24252, Korea
}

\begin{abstract}
The serine/threonine kinase mTOR is essential for the phosphoinositide 3-kinases ( $\mathrm{PI} 3 \mathrm{~K}$ ) signaling pathway, and regulates the development and function of immune cells. Aberrant activation of mTOR signaling pathway is associated with many cancers including leukemia. Here, we report the contributions of mTOR signaling to growth of human leukemic cell lines and mouse T-cell acute leukemia (T-ALL) cells. Torin, an ATP-competitive mTOR inhibitor, was found to have both cytotoxic and cytostatic effects on U-937, THP-1, and RPMI-8226 cells, but not on Jurkat or K-562 cells. All cells were relatively resistant to rapamycin even with suppressed activity of mTOR complex 1. Growth of T-ALL cells induced by Notch1 was profoundly affected by torin partially due to increased expression of $B c / 2 / 11$ and Bbc3. Of note, activation of Akt or knockdown of FoxO1 mitigated the effect of mTOR inhibition on T-ALL cells. Our data provide insight on the effect of mTOR inhibitors on the survival and proliferation of leukemic cells, thus further improving our understanding on cell-context-dependent impacts of mTOR signaling. [BMB Reports 2016; 49(1): 63-68]
\end{abstract}

\section{INTRODUCTION}

The serine/threonine kinase mTOR plays a central role in the phosphoinositide 3-kinases (PI3K) signaling pathway, and regulates the development and function of immune cells (1). mTOR exists in two multiprotein complexes: mTORC1 and mTORC2. They have distinct regulatory subunits with different substrate specificities (2). Rapamycin-sensitive mTORC1 is essential for the growth and proliferation of lymphoid cells by upregulating the translation, lipid synthesis, and metabolism while suppressing autophagy $(2,3)$. On the other hand, mTORC2 is largely insensitive to rapamycin. It phosphorylates hydrophobic motifs (HM) of Akt, SGK1, and PKC isoforms,

*Corresponding author. Tel: +82-33-248-2113; Fax: +82-33-2563420; E-mail: keunwook@hallym.ac.kr

http://dx.doi.org/10.5483/BMBRep.2016.49.1.201

Received 7 October 2015, Revised 19 October 2015, Accepted 22 October 2015

Keywords: mTOR, torin, rapamycin, leukemic cell, T-ALL which is important for the proliferation, cytoskeletal organization, and differentiation $(2,4,5)$. Recent studies using tissuespecific conditional $\mathrm{KO}$ mice have revealed distinct roles of mTOR complexes in the development and function of immune cells, including $T$ cells, B cells, macrophages, and dendritic cells $(1,5-8)$.

Malignant transformation and progression of cancer depend on sustained activation of trophic signaling pathways. Aberrant activation of $\mathrm{PI} 3 \mathrm{~K} / \mathrm{Akt} / \mathrm{mTOR}$ signaling pathway is closely associated with many types of cancers, including hematologic malignancies $(9,10)$. For example, constitutive activation of Akt has been reported in more than $60 \%$ of acute myelogenous leukemia and up to $88 \%$ of acute lymphoblastic leukemia (ALL) $(11,12)$. Tumor suppressor Pten negatively controls PI3K signaling. Conditional deletion of Pten from mouse hematopoietic compartment is sufficient to cause acute T cell leukemia and myeloid proliferative disorder (13). Intriguingly, depletion of a regulatory subunit from either mTORC1 or mTORC2 can dramatically attenuate mouse leukemogenesis triggered by Pten loss $(13,14)$. Furthermore, inactivation of either mTORC1 or mTORC2 can reduce mouse mortality of T-cell acute lymphoblastic leukemia (T-ALL) evoked by constitutive activation of Notch1 $(6,8)$. These evidences suggest that mTOR is an attractive target for leukemia treatment. Allosteric mTOR inhibitor rapamycin and its analogues have been clinically tested for several types of cancers (10). In contrast to the impact of genetic ablation of mTORC1 in the leukemic mouse models, rapamycin has relatively modest effect on the growth and proliferation of B-cell precursor ALL and acute myeloid leukemia (AML) cells $(15,16)$. This might be due to increased Akt activity as a negative feedback regulation of $\mathrm{mTORC1}$, and/or due to incomplete inhibition of rapamycin depending on cell type $(17,18)$. Prolonged treatment of rapamycin can suppress Akt activation by inhibiting mTORC2 in some cell lines and primary T cells $(4,19)$. A new class of ATP competitive mTOR inhibitors has been developed to overcome the limitation of rapamycin by potentially targeting both mTOR complexes. For example, torin, an active-site mTOR inhibitor, is potent in suppressing both mTORC1 and mTORC2 activities, and effective in inhibiting the growth of several ALL cell lines $(16,20)$.

The objective of this study was to determine the susceptibility of several leukemic cell lines to rapamycin and torin, and assess the contribution of mTOR signaling to the growth of

ISSN: 1976-670X (electronic edition)

Copyright (c) 2016 by the The Korean Society for Biochemistry and Molecular Biology

(c) This is an open-access article distributed under the terms of the Creative Commons Attribution Non-Commercial License (http://creativecommons.org/licenses/by-nc/4.0) which permits unrestricted non-commercial use, distribution, and reproduction in any medium, provided the original work is properly cited. 
leukemic cells using mTOR inhibitors. The survival and proliferation of human leukemic cell lines were markedly affected by dual mTOR inhibitor torin, although some cells were less sensitive. On the other hand, rapamycin exhibited relative modest cytostatic effects on leukemic cell lines without inducing apoptosis. Using Notch1-driven mouse primary T-ALL cells, we demonstrated that rapamycin-resistant and torin-sensitive mTOR activity was crucial for the persistence of T-ALL cells. Furthermore, using modification of mTOR signaling components, our results suggest that targeting mTORC2/Akt/FoxO signaling pathway could be a promising strategy for treating T-ALL.

\section{RESULTS}

\section{Effect of mTOR inhibitors on the survival and proliferation of} human leukemic cell lines

mTOR signaling regulates the growth, proliferation, and function of normal immune cells in a cell-dependent manner (1, 4, 5). To define the roles of mTOR activity on the growth and maintenance of leukemic cells, we compared the impact of two mTOR inhibitors: mTOR allosteric inhibitor rapamycin and active-site inhibitor torin. Human leukemic cell lines were cultured in the presence of these inhibitors and cell death was
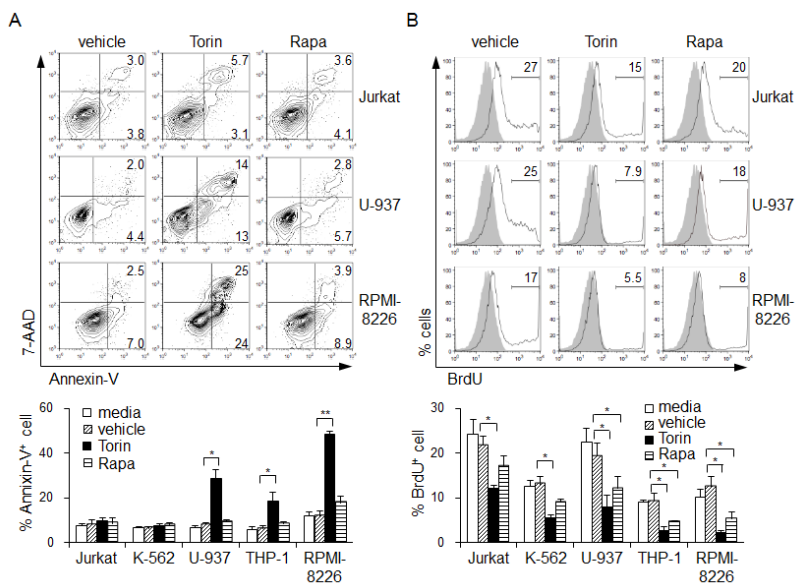

Fig. 1. Effect of mTOR inhibitors on the survival and proliferation of leukemic cells. (A) Human leukemic cell lines were cultured for $18 \mathrm{~h}$ in the presence of $50 \mathrm{nM}$ rapamycin or $250 \mathrm{nM}$ torin and stained with Annexin- $V$ and 7-AAD. Representative FACS profiles from four independent experiments are shown. Numbers denote the percentage of $7-\mathrm{AAD}^{+}$Annexin- $\mathrm{V}^{+}$and 7-AAD ${ }^{-}$Annexin- $\mathrm{V}^{+}$cells, respectively. (B) Cells were cultured as described in (A) and assayed for BrdU uptake as described in "MATERIALS AND METHODS". Histograms for the signal of no BrdU controls (gray) or BrdU-pulsed samples (line) representing three independent experiments are shown. Numbers indicate the percentage of $\mathrm{BrdU}^{+}$cells. Experimental results are summarized in the bottom panels as mean $( \pm$ SEM) percentages of Annexin- $\mathrm{V}^{+}$(A) and BrdU $^{+}$(B) cells, respectively: $* P<0.05 ; * * P<0.01$. examined by staining cell surface Annexin-V (Fig. 1A). Torin treatment resulted in apoptosis of monocyte-derived leukemic cell lines U-937 and THP-1. However, rapamycin exhibited no cytotoxic activity against these leukemic cells. Interestingly, myeloma-derived RPMI-8226 cells were highly sensitive to torin, whereas Jurkat (Pten mutant T-ALL cell line) and K-562 $\left(\mathrm{Bcr}-\mathrm{Abl}^{+} \mathrm{AML}\right.$ cell line) cells were resistant to torin (Fig. 1A). It is known that the progression and maintenance of leukemia depend on sustained proliferative signaling (9). When cells were pulsed with bromodeoxyuridine (BrdU) for $8 \mathrm{~h}, 11-25 \%$ of leukemic cells were $\mathrm{BrdU}^{+}$cells, indicating the progression of $S$ phase of the cell cycle (Fig. 1B). Torin treatment substantially decreased BrdU uptake in all cell lines tested. However, rapamycin had relatively modest but significant cytostatic effects on U-937, THP-1, and RPMI-8226 cells, but not on Jurkat or K-562 cells (Fig. 1B). These results indicated that mTOR activity was important for the survival and proliferation of leukemic cells, illustrating a leukemic cell-dependent function of mTOR signaling.

To determine the impact of torin and rapamycin on mTOR signaling, we measured the phosphorylation levels of proteins downstream of mTORC1 and mTORC2. The activity of mTORC1 was highly sensitive to both inhibitors based on impaired phosphorylation of S6K and S6, although the phosphorylation of $4 \mathrm{E}-\mathrm{BP} 1$ was only partially suppressed by rapamycin (Supplemental Fig. S1a). Treatment with rapamycin for $18 \mathrm{~h}$ was not sufficient enough to inhibit the activity of mTORC2 that it did not affect the HM phosphorylation of Akt (S473) in all leukemic cell lines except Jurkat cells. In accordance with results of previous studies (19), prolonged treatment of rapamycin was able to suppress HM phosphorylation of Akt in Jurkat cells without affecting the survival or proliferation signaling (Fig. 1 \& Supplemental Fig. S1a). In contrast, torin efficiently inhibited the HM phosphorylation of Akt in all cell lines tested. mTORC2 is reported to be able to phosphorylate the HM of PKC isoforms and SGK1 $(2,5)$. When cells were treated with torin, HM phosphorylation of PKC ( $\beta$ IIS660) was decreased in Jurkat cells but not in U-937 cells. In addition, HM phosphorylation of NDRG1, a downstream target of SGK1, was partially decreased in U-937 cells but not in Jurkat cells (Supplemental Fig. S1a).

$\mathrm{Akt} / \mathrm{mTOR}$ signaling regulates the expression of $\mathrm{Bcl}-2$ family genes, which determines cell viability in a mitochondria-dependent manner (5). The expression levels of pro-survival genes such as $B C l 2$ and $B C l 2 / 1$ (encoding $B C l-X_{L}$ ) were not decreased by the two mTOR inhibitors (Supplemental Fig. S1b). Instead, torin treatment significantly upregulated the expression of pro-apoptotic genes such as Bcl2/11 (Bim) and Cdkn1b $\left(\mathrm{p} 27^{\mathrm{Kip} 1}\right)$ in U-937, THP-1, and RPMI-8226 cells, but not in K-562 cells. Together with different cytotoxicity of torin on leukemic cell lines, these results imply that rapamycin-resistant and torin-sensitive mTOR signaling is associated with Bcl-2-dependent apoptosis in leukemic cells. 
Effect of mTOR inhibitors on the survival and proliferation of Notch-driven mouse leukemic cells

Although mTORC1-restricted inhibition with rapamycin had a marginal effect, dual mTOR inhibitor torin efficiently suppressed the proliferation and/or the survival of human leukemic cells. To test the impact of the two mTOR inhibitors on primary leukemic cells, we employed a Notch1-driven mouse T-ALL. Bone marrow cells were retrovirally transduced with ICN1, a constitutively-active form of Notch1, and transplanted into irradiated recipient mice. Primary T-ALL cells were isolated from leukemic mice and maintained on OP9 stromal cells in the presence of IL-7. As shown in Fig. 2A, at 6 days after co-culturing with OP9 cells, all cells expressed T-lineage marker Thy1.2, with more than $95 \% \mathrm{GFP}^{+}$as a surrogate for ICN1 expression. When cells were cultured in the presence of mTOR inhibitors, the persistence of T-ALL cells was severely impaired (Fig. 2A, right). Torin treatment resulted in increased cell surface expression of Annexin- $V$ but suppressed BrdU uptake (Fig. 2B \& C), revealing that torin was cytotoxic and cytostatic to T-ALL cells. Intriguingly, treatment with rapamycin al-

A
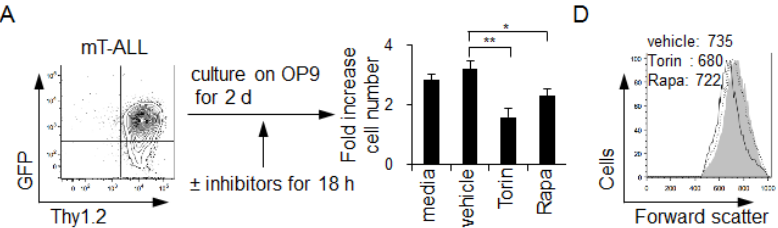

B
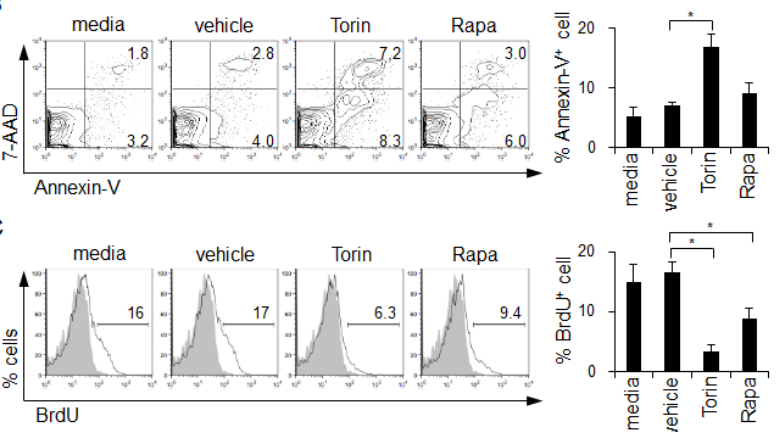

Fig. 2. Effect of mTOR inhibitors on Notch-driven mouse leukemic cells. (A) Primary T-ALL cells were generated by transducing bone marrow cells with a constitutively-active form of Notch1 as described in "MATERIALS AND METHODS" and examined by flow cytometry (left) as a quality control. Cells were treated with mTOR inhibitors in the presence of OP9 stromal cells. Viable lymphoblastic cells were counted by trypan-blue dye exclusion. Means $( \pm$ SEM) for T-ALL cell expansion in the culture (fold increase $=$ cells recovered $/$ input cells) are shown in the right. (B \& C) T-ALL cells were treated with rapamacyin or torin for $18 \mathrm{~h}$ and assayed for apoptosis and proliferation as in Fig. 1. Representative FACS profiles (left panels) from four independent experiments are shown. Mean $\left( \pm\right.$ SEM) percentages of Annexin- $\mathrm{V}^{+}$or BrdU ${ }^{+}$cells are shown in the right panels. (D) Histograms of forward scattering with mean light intensity (MLI) values. Shaded: vehicle treated; solid line: torin-treated; dashed line: rapamycin-treated. so impaired the persistence of T-ALL cells due to impaired proliferation instead of survival defect of T-ALL cells. Although mTORC1 was known to be able to regulate cell growth and metabolism in lymphocytes (3), rapamycin-treated T-ALL cells and vehicle-treated cells were comparable in cell sizes (forward scattering), whereas torin-treated cells were smaller (Fig. 2D).

When T-ALL cells were treated with rapamycin for $18 \mathrm{~h}$, the phosphorylation levels of S6K and S6 were substantially impaired and that of 4E-BP1 was modestly decreased (Fig. 3A). Similar to results obtained from human leukemic cell lines except Jurkat cells, prolonged treatment with rapamycin was not sufficient enough to inhibit mTORC2 activities based on HM phosphorylation of Akt. On the other hand, torin efficiently inhibited both mTORC1 and mTORC2 activities in mouse T-ALL cells (Fig. 3A). For the downstream targets of mTORC2, torin exerted a potent inhibitory effect on HM phosphorylation of Akt, but not on that of PKC. Consistent with reduced HM phosphorylation of Akt, the phosphorylation of Akt substrate FoxO1/3a was decreased in torin-treated T-ALL cells (Fig. 3A). Moreover, the phosphorylation of NDRG1, a downstream target of SGK1, was also affected by torin treatment. These results revealed that torin and rapamycin had different effects on

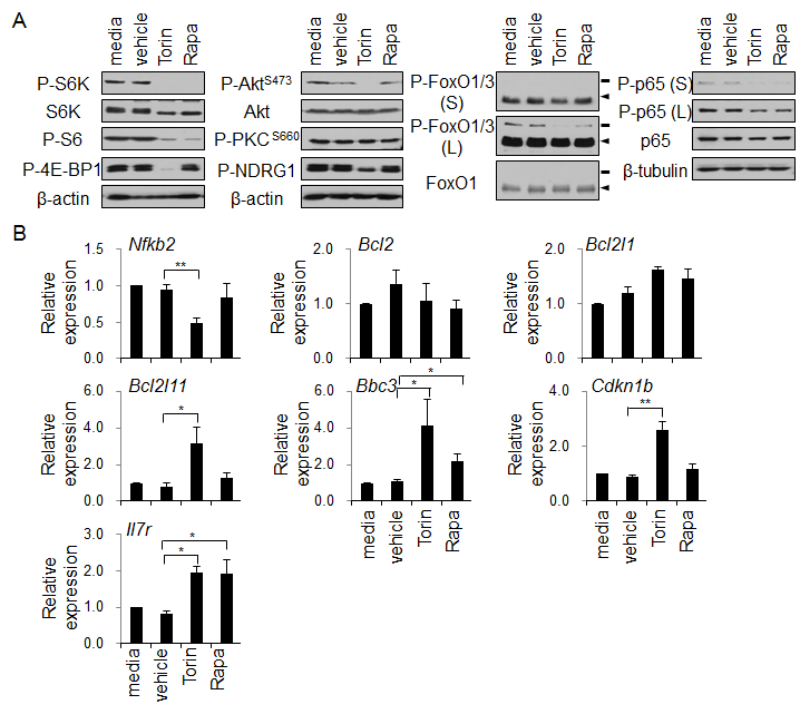

Fig. 3. mTOR modulates signaling and expression of genes associated with survival and proliferation of T-ALL cells. (A) Mouse T-ALL cells were cultured on OP9 cells in the presence or absence of mTOR inhibitors for $18 \mathrm{~h}$ as described in Fig. 2 and analyzed by immunoblots with the indicated antibodies. This is a representative of two or three independent experiments. Arrowheads indicate P-FoxO1. Lines indicate P-FoxO3a. $\beta$-actin and $\beta$-tubulin were used as loading controls. (B) T-ALL cells were treated with rapamycin or torin for $8 \mathrm{~h}$ and subjected to real-time reverse transcriptional PCR. Means ( \pm SEM) of expression for the indicated mRNA relative to that of untreated control sample (media) were obtained from three or four independent experiments. 
mTOR activities, indicating the importance of rapamycin-resistant and torin-sensitive mTOR signaling in the persistence of T-ALL cells.

Transcription factor NF- $\mathrm{kB}$ can regulate the expression of a wide array of genes associated with cell survival and cell cycling. Previously, we and other groups have reported that mTOR signaling can promote NF-kB activation via Akt or IKK $(6,21)$. In this study, our results revealed that mTOR inhibition by torin and rapamycin resulted in impaired phosphorylation of NF-kB p65 in T-ALL cells (Fig. 3A). Although mTOR inhibition reduced the expression of $N f k b 2$, a well-known target

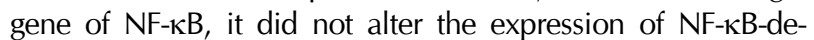
pendent pro-survival genes such as $B C l 2$ and $B C l 2 / 1$ (Fig. 3B). Instead, NF-kB-responsive pro-apoptotic gene $B b c 3$ (encoding PUMA) (22) was highly expressed in torin-treated T-ALL cells compared to that in the controls. In accordance with impaired phosphorylation of FoxO1/3a, the expression of FoxO target gene $B C / 2 / 11$ (encoding Bim) was augmented in torin-treated cells. In addition, the expression of $C d k n 1 b$, a target gene of p53, was also increased in torin-treated cells (Fig. 3B). These results suggest that torin-dependent mTOR signaling can negatively regulates the induction of pro-apoptotic genes such as $B b c 3$ and $B c / 2 / 11$ possibly by modulating NF- $\mathrm{KB}$ and FoxO transcription factors, respectively. As we supplemented T-ALL cell with IL-7 and measured the mRNA expression of IL-7 receptor, we did not observe diminished expression of $1 / 7 r$ in

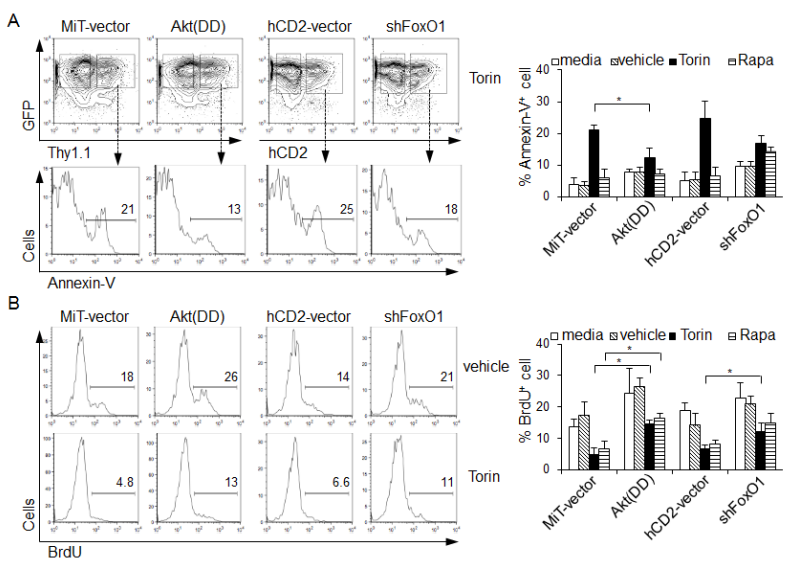

Fig. 4. Activation of Akt and knockdown of FoxO1 mitigates the effect of torin on T-ALL cells. Mouse T-ALL cells were retrovirally transduced with Akt(DD), shFoxO1, or control vectors (MiT-vector or hCD2-vector) and cultured on OP9 stromal cells in the presence or absence of mTOR inhibitors. Cells were stained with anti-Thy1.1 or anti-hCD2 antibodies (upper panels in A). Apoptosis and proliferation were measured by Annexin- $\mathrm{V}$ staining (A) and BrdU incorporation assay (B), respectively. Representative histograms of Annexin- $V$ (bottom panels in $A$ ) or BrdU uptake (B) in the transduced cell $\left(\right.$ Thy $1.1^{+}$or $\mathrm{hCD} 2^{+}$) gates are shown. Mean $( \pm \mathrm{SEM})$ percentages of Annexin- $\mathrm{V}^{+}$or $\mathrm{BrdU}^{+}$cells were obtained from three independent experiments and summarized in the right panels. torin-treated cells (Fig. 3B).

\section{Akt/FoxO signaling promotes the growth of T-ALL cells}

Torin treatment inhibited mTORC2-induced activation of Akt and the resulting phosphorylation of its downstream target FoxO transcription factors in T-ALL cells. To further explore the functional implication of $\mathrm{Akt} / \mathrm{FoxO}$ signaling in the survival and proliferation of T-ALL cells, we transduced cells with Akt(DD) [Akt T308D, S473D], an active mutant of Akt, or shFoxO1. Ectopic expression of Akt(DD) rescued torin-treated T-ALL cells from apoptosis and cell cycle inhibition, rendering them similar to vehicle-treated cells transduced with control vector (Fig. 4A, B). Transduction with Akt(DD) also significantly increased the proliferation of rapamycin-treated T-ALL cells (Fig. 4B). Akt-dependent phosphorylation of FoxO transcription factors can lead to the translocation of FoxO out of nucleus, thus promoting cell survival and cell cycling (23). Knockdown of FoxO1 using shFoxO1 transduction partially restored BrdU uptake of T-ALL cells treated with mTOR inhibitors. However, it failed to rescue them from apoptosis (Fig. 4A, B). Overall, our results suggest that Akt/FoxO signaling downstream from mTORC2 is crucial for the survival and proliferation of T-ALL cells. This signaling pathway provides a potent therapeutic strategy with better efficacy for leukemia treatment.

\section{DISCUSSION}

Conditional deletion of mTOR subunits in hematopoietic compartment causes significant thymic atrophy due to impaired growth and proliferation of pre-T cells $(6,8)$. In particular, both mTORC1 and mTORC2 are vital for the development of mouse T-cell leukemia induced by Notch1, whose mutations have been identified in more than $50 \%$ of T-ALL patients (10). In this study, we determined the effect of mTOR inhibitors on the persistence of leukemic cells and investigated a relative contribution of mTOR signaling components to the survival and proliferation of T-ALL cells. Using human leukemic cell lines, we observed cell-dependent sensitivity to torin and rapamycin. A further study is required to clarify what can cause different sensitivity of leukemic cells to mTOR inhibition. While mTORC1-restricted inhibition using rapamycin modestly attenuated cell proliferation without affecting cell survival, torin exhibited cytotoxic and cytostatic effect via suppressing both mTORC1 and mTORC2 activities. We recapitulated this distinct impact of mTOR inhibitors on mouse T-ALL cells was driven by Notch1 and found that rapamycin-resistant and torin-sensitive mTOR signaling was crucial for the survival of T-ALL cells.

Of note, the expression levels of pro-apoptotic genes such as $B C l 2 / 11$ and $B b c 3$ as well as p53 target gene $C d k n 1 b$ were markedly upregulated by torin treatment. Based on these results, mTORC2 signaling and/or rapamycin-resistant mTORC1 activity may have a role in the regulation of genes associated 
with apoptosis and cell cycle arrest via modulating FoxOs and NF-kB. Transduction of T-ALL cells with Akt (DD) accelerated the proliferation of torin-treated T-ALL cells but suppressed apoptosis induced by torin treatment (Fig. 4). However, the connection between Akt and NF-kB is still under debate. Although knockdown of FoxO1 alone was not sufficient enough to suppress apoptosis, treatment with torin rescued cells from cell cycle arrest, suggesting that Akt/FoxO signaling components is associated with the proliferation of T-ALL cells. Central challenges facing targeted therapy for T-ALL include toxicities to hematopoietic stem cells (HSCs). In fact, inactivation of mTORC1 can severely impair the maintenance and function of HSCs, whereas mTORC2 appears to be dispensable $(13,14)$. Thus, targeting rapamycin-resistance and torin-sensitive mTOR signaling components might offer a novel therapeutic approach with lower inherent side effects for leukemia treatment.

\section{MATERIALS AND METHODS}

\section{Cell lines and reagents}

U-937, THP-1, K-562, and RPMI-8226 cells were obtained from Hyung-Joo Kwon (Hallym University). Jurkat cells were obtained from Mark Boothby (Vanderbilt University). Human leukemic cell lines were maintained with complete RPMI1640 medium containing $10 \%$ fetal bovine serum (FBS) at $37^{\circ} \mathrm{C}$ supplied with $5 \% \mathrm{CO}_{2}$. Torin-2 and rapamycin were purchased from Tocris Bioscience. Recombinant murine IL-3, IL-6, IL-7, and SCF were obtained from Peprotech Inc.. 5-fluorouracil (5-FU) was purchased from Sigma-Aldrich. PE-conjugated Annexin- $V$ and fluorophore-conjugated antibodies were purchased from BD Bioscience or eBioscience.

\section{ICN1-transduced mouse T-ALL cells}

C57BL/6 mice were purchased from Orient Bio and housed in specific pathogen-free conditions. Experimental protocols were approved by the Institutional Animal Care and Use Committee of Hallym University. Mouse T-ALL cells were prepared as described previously (6). Briefly, bone marrow cells were isolated from mice at $4 \mathrm{~d}$ after treatment with $150 \mathrm{mg} / \mathrm{Kg} \mathrm{5-FU}$ and incubated in complete IMDM containing 15\% FBS, 10 $\mathrm{ng} / \mathrm{ml} \mathrm{IL-3,} 10 \mathrm{ng} / \mathrm{ml} \mathrm{IL-6}$, and $50 \mathrm{ng} / \mathrm{ml} \mathrm{SCF}$ for $2 \mathrm{~d}$. Cells were retrovirally transduced with MSCV-ICN1-IRES-GFP and injected intravenously into a lethally irradiated syngeneic recipient along with equal amount $\left(5 \times 10^{5}\right.$ cells) of unmanipulated bone marrow cells. Leukemic cells were obtained from lymph nodes of leukemic mice at 4 weeks post-transplantation and maintained on OP9 stromal cells in IMDM containing $10 \%$ FBS supplemented with $5 \mathrm{ng} / \mathrm{ml} \mathrm{IL-7.}$

\section{Annexin- $V$ staining}

Cells were cultured in the presence or absence of mTOR inhibitors for $18 \mathrm{~h}$ and stained with PE-conjugated Annexin-V and 7-AAD (Invitrogen) in $20 \mathrm{mM}$ HEPES (pH 7.4), $140 \mathrm{mM}$
$\mathrm{NaCl}$, and $2.5 \mathrm{mM} \mathrm{CaCl}_{2}$. Retrovirally transduced cells were stained with surface markers (PE-conjugated anti-Thy1.1 or PerCP-conjugated anti-hCD2) along with Annexin- $\mathrm{V}$ and 7-AAD. Data was acquired using a BD FACSCanto-2 or FACSCalibur. The incidence of Annexin- $\mathrm{V}^{+}$apoptotic cells was analyzed using Flowjo (Treestar).

\section{BrdU incorporation assay}

For cell proliferation assays, cells were cultured with vehicle (0.001\% DMSO), $250 \mathrm{nM}$ torin, or $50 \mathrm{nM}$ rapamycin for $10 \mathrm{~h}$, followed with the addition of $10 \mu \mathrm{M}$ 5-bromo-2-deoxyuridine (BrdU) to the culture and cultured for $8 \mathrm{~h}$. $\mathrm{S}$ phase entry rates were determined with anti-BrdU staining and flow cytometry as described previously (5). Briefly, cells were fixed and permeabilized using BrdU staining buffer set (eBioscience), treated with DNase (Sigma-Aldrich) for $1 \mathrm{~h}$ at $37^{\circ} \mathrm{C}$, and then stained with APC-conjugated anti-BrdU antibody. Retrovirally transduced cells were stained with surface markers followed by BrdU staining.

\section{Retroviral transduction}

Retroviral constructs of MSCV-Akt(DD)-IRES-Thy1.1 [MiT-Akt (DD)], MSCV-shFoxO1-IRES-hCD2 [hCD2-shFoxO1], and control vectors (MiT-vector and hCD2-vector) were used as described previously (6). Retrovirions were collected from culture supernatant of phoenix packaging cells transfected with indicated plasmids and centrifuged $(2,500 \mathrm{~g})$ with mouse T-ALL cells for spinfection. After $1 \mathrm{~h}$ of spinfection, T-ALL cells were co-cultured on OP9 stromal cells supplemented with 5 $\mathrm{ng} / \mathrm{ml}$ of IL-7.

\section{Westem blotting and measurement of RNA levels}

Cells were lysed in $20 \mathrm{mM}$ HEPES ( $\mathrm{pH} 7.4), 150 \mathrm{mM} \mathrm{NaCl}$, $1 \%$ Triton X-100, 1 mM EDTA, protease, and phosphatase inhibitors (GenDEPOT). Proteins were extracted and subjected to SDS-PAGE. Immunoblotting was performed using antibodies against P-Akt ${ }^{\mathrm{S473}}, \mathrm{P}-\mathrm{PKC}\left(\beta_{\|} \mathrm{S} 660\right), \mathrm{P}-\mathrm{NDRG} 1^{\mathrm{T} 346}, \mathrm{P}-\mathrm{S} 6 \mathrm{~K}^{\mathrm{T} 389}$, $\mathrm{P}-\mathrm{S} 6^{\mathrm{S} 240 / 244}, \mathrm{P}-4 \mathrm{E}-\mathrm{BP} 1^{\mathrm{T} 0}, \mathrm{P}-\mathrm{FoxO} 1^{\mathrm{T} 24} / 3 \mathrm{a}^{\mathrm{T} 2}{ }^{2}, \mathrm{Akt}$, FoxO1, $\beta$-tubulin, and $\beta$-actin. All antibodies were purchased from Cell Signaling Technologies except S6K (Santa Cruz Biotechnology) and $\beta$-actin (Sigma-Aldrich). Total RNAs were isolated using Trizol (Invitrogen). cDNAs were synthesized with MMLV reverse transcriptase (Promega) using random nonamers. RNA levels were measured with quantitative real-time PCR using gene specific prime pairs (Supplemental Table S1) and a CFX Real-Time PCR Detection System (Bio-Rad).

\section{Statistics}

All experiments were performed in duplicates or triplicates. Data were presented as mean \pm SEM from three independent experiments. Difference between samples were analyzed statistically using unpaired two-tailed nonparametric t-test. Symbols $*$ and ${ }^{* *}$ indicated statistical significance at $p$ values less than 0.05 and 0.01 , respectively. 


\section{ACKNOWLEDGEMENTS}

This research was supported by a grant (NRF-2014R1A1A4A01008734) of Basic Science Research Program funded by the Ministry of Education through the National Research Foundation, a Korean Mouse Phenotyping Project (NRF-2014M3A9D5A01073841) funded by the Ministry of Science, ICT, and Future Planning through the National Research Foundation, and Hallym University Research Fund 2014 (H20140034).

\section{REFERENCES}

1. Powell JD, Pollizzi KN, Heikamp EB and Horton MR (2012) Regulation of immune responses by mTOR. Annu Rev Immunol 30, 39-68

2. Laplante M and Sabatini DM (2012) mTOR signaling in growth control and disease. Cell 149, 274-293

3. Zeng $H$ and Chi $H$ (2013) mTOR and lymphocyte metabolism. Curr Opin Immunol 25, 347-355

4. Lee K, Gudapati P, Dragovic S et al (2010) Mammalian target of rapamycin protein complex 2 regulates differentiation of Th1 and Th2 cell subsets via distinct signaling pathways. Immunity 32, 743-753

5. Lee K, Heffington L, Jellusova J et al (2013) Requirement for Rictor in homeostasis and function of mature B lymphoid cells. Blood 122, 2369-2379

6. Lee K, Nam KT, Cho SH et al (2012) Vital roles of mTOR complex 2 in Notch-driven thymocyte differentiation and leukemia. J Exp Med 209, 713-728

7. Weichhart T, Hengstschlager $M$ and Linke $M$ (2015) Regulation of innate immune cell function by mTOR. Nat Rev Immunol 15, 599-614

8. Hoshii T, Kasada A, Hatakeyama T et al (2014) Loss of mTOR complex 1 induces developmental blockage in early T-lymphopoiesis and eradicates T-cell acute lymphoblastic leukemia cells. Proc Natl Acad Sci U S A 111, 3805-3810

9. Engelman JA (2009) Targeting PI3K signalling in cancer: opportunities, challenges and limitations. Nat Rev Cancer 9, 550-562

10. Bertacchini J, Heidari N, Mediani L et al (2015) Targeting $\mathrm{PI} 3 \mathrm{~K} / \mathrm{AKT} / \mathrm{mTOR}$ network for treatment of leukemia. Cell Mol Life Sci 72, 2337-2347

11. Tamburini J, Elie C, Bardet V et al (2007) Constitutive phosphoinositide 3-kinase/Akt activation represents a fa- vorable prognostic factor in de novo acute myelogenous leukemia patients. Blood 110, 1025-1028

12. Silva A, Yunes JA, Cardoso BA et al (2008) PTEN posttranslational inactivation and hyperactivation of the PI3K/Akt pathway sustain primary T cell leukemia viability. J Clin Invest 118, 3762-3774

13. Magee JA, Ikenoue T, Nakada D, Lee JY, Guan KL and Morrison SJ (2012) Temporal changes in PTEN and mTORC2 regulation of hematopoietic stem cell self-renewal and leukemia suppression. Cell Stem Cell 11, 415-428

14. Kalaitzidis D, Sykes SM, Wang Z et al (2012) mTOR complex 1 plays critical roles in hematopoiesis and Pten-lossevoked leukemogenesis. Cell Stem Cell 11, 429-439

15. Xu Q, Thompson JE and Carroll M (2005) mTOR regulates cell survival after etoposide treatment in primary $\mathrm{AML}$ cells. Blood 106, 4261-4268

16. Simioni C, Cani A, Martelli AM et al (2014) Activity of the novel mTOR inhibitor Torin-2 in B-precursor acute lymphoblastic leukemia and its therapeutic potential to prevent Akt reactivation. Oncotarget 5, 10034-10047

17. Wan X, Harkavy B, Shen N, Grohar P and Helman LJ (2007) Rapamycin induces feedback activation of Akt signaling through an IGF-1R-dependent mechanism. Oncogene 26, 1932-1940

18. Feldman ME, Apsel B, Uotila A et al (2009) Active-site inhibitors of mTOR target rapamycin-resistant outputs of mTORC1 and mTORC2. PLoS Biol 7, e38

19. Sarbassov DD, Ali SM, Sengupta S et al (2006) Prolonged rapamycin treatment inhibits mTORC2 assembly and Akt/PKB. Mol Cell 22, 159-168

20. Badura S, Tesanovic T, Pfeifer $\mathrm{H}$ et al (2013) Differential effects of selective inhibitors targeting the PI3K/AKT/mTOR pathway in acute lymphoblastic leukemia. PLoS One 8, e80070

21. Dan HC, Cooper MJ, Cogswell PC, Duncan JA, Ting JP and Baldwin AS (2008) Akt-dependent regulation of NF\{kappa\}B is controlled by mTOR and Raptor in association with IKK. Genes Dev 22, 1490-1500

22. Wang P, Qiu W, Dudgeon C et al (2009) PUMA is directly activated by NF-kappaB and contributes to TNF-alpha-induced apoptosis. Cell Death Differ 16, 1192-1202

23. Brunet A, Bonni A, Zigmond MJ et al (1999) Akt promotes cell survival by phosphorylating and inhibiting a Forkhead transcription factor. Cell 96, 857-868 